\title{
Imaging of Biologically Derived Anisotropic Fluids Using Environmental Scanning Electron Microscopy
}

\author{
A.F. Miller and A.M. Donald \\ Polymer and Colloid Group, Cavendish Laboratory, University of Cambridge, Cambridge, CB3 \\ OHE.
}

Environmental scanning electron microscopy (ESEM) provides a novel, high spacial resolution technique to study the surface organisation of anisotropic solutions. We have investigated various categories of biopolymers and here we will outline the characteristics of one model system: aqueous suspensions of rod-like cellulose crystallites.

Such cellulose microcrystalline suspensions have been prepared by acid hydrolysis of natural cellulose fibrils from various sources ${ }^{1}$. It has been found that in a limited concentration range such suspensions separate spontaneously into a chiral nematic (cholesteric) phase and an isotropic phase (organisation of each phase is outlined schematically in figure 1) ${ }^{2,3}$. By manipulating the treatment conditions the quantity of surface charge introduced to the material has been controlled from zero upwards (quantified using conductometric titration). Transmission electron micrographs reveal that the microscopic size and shape of the cellulose rods are the same irrespective of the preparation method but the level of surface charge, concentration and ionic strength has been shown to influence the physical properties of the system, for example aggregation and viscosity. Phase diagrams outlining such behaviour will be presented and discussed in relation to theoretical predictions for rod-like particles.

The organisation of the self-assembled structures has been investigated using both polarising microscopy and ESEM and results obtained are comparable ${ }^{4}$. Polarising microscopy studies revealed the lower of the two phases displayed liquid crystalline characteristics where nematic 'tactoids' were initially viewed (figure 2a) but these equilibrated over time (few hours to two days) to produce textures indicative of helicoidally oriented chiral nematic (cholesteric) liquid crystalline phases (figure 2b). The upper, less dense, phase was isotropic. Micrographs of the isotropic phase obtained using ESEM show that individual cellulose chains aggregate along their chain length, presumably through electrostatic interaction and hydrogen bonding, to form fibrils circa $200 \mathrm{~nm}$ in length and 5 $\mathrm{nm}$ wide that lie in the surface plane. A typical ESEM micrograph of the 'tactoids' formed from the anisotropic phase is given in figure 3. High resolution imaging revealed that the 'tactoids' consist of individual fibrils aligning along their length and have a preferred, in plane orientation. Imaging the equilibrium anisotropic structures involved leaving a few drops of fluid on the sample stub, in a saturated water environment (to avoid evaporation) for 2-3 hours, before placing into the ESEM sample chamber. A typical image obtained is given in figure $3 \mathrm{~b}$ where fingerprint patterns characteristic of the cholesteric phase are evident. It is believed that this is the first observation of a liquid crystalline texture in the ESEM. Supporting evidence for this hypothesis comes from analysis of the cholesteric pitch, $\mathrm{P}$, of the anisotropic phase. The pitch is a measure of cellulose interactions (smaller pitch, stronger interaction) and is determined by measuring the distance between fingerprint lines ${ }^{4}$. Both techniques gave comparable results, both qualitatively and quantitatively, where the pitch decreased with both increasing concentration and ionic strength. Further micrographs and 
possible electron image contrast mechanisms will be discussed in this paper. The structural information obtained will be correlated to surface and interfacial tension values.

References

[1] J.F. Revol et al. Int. J. Biol. Macromol. 14 (1992) 170.

[2] J.F. Revol et al., Liq. Crys. 16 (1994) 127.

[3] X.M. Dong et al. Langmuir 12 (1996) 2076.

[4] A.F. Miller and A.M. Donald, Submitted to Langmuir (2002).
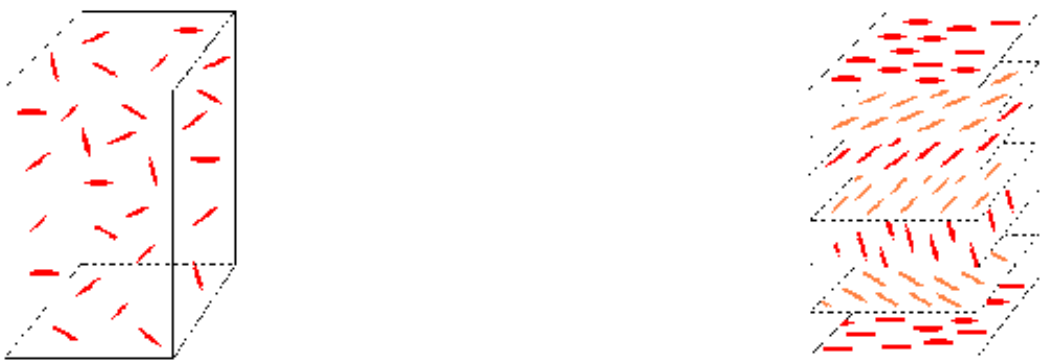

FIG. 1. Schematic representation of a) isotropic and b) cholesteric phases.
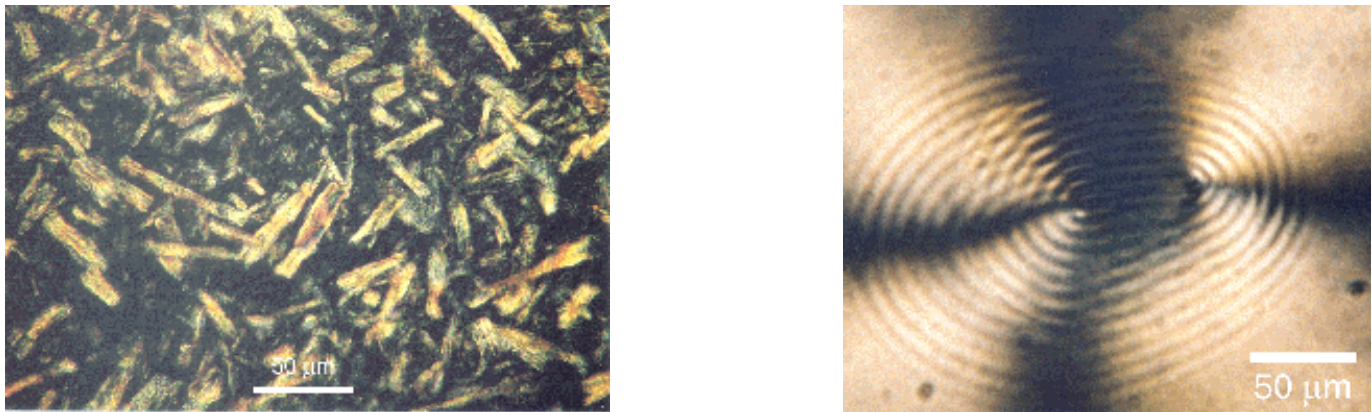

FIG. 2. Optical micrographs of liquid crystalline phase texture a) minutes and b) hours after slide preparation (Beam energy was $10 \mathrm{keV}$, chamber pressure 4.0 Torr and sample temperature 275 $\mathrm{K})$.
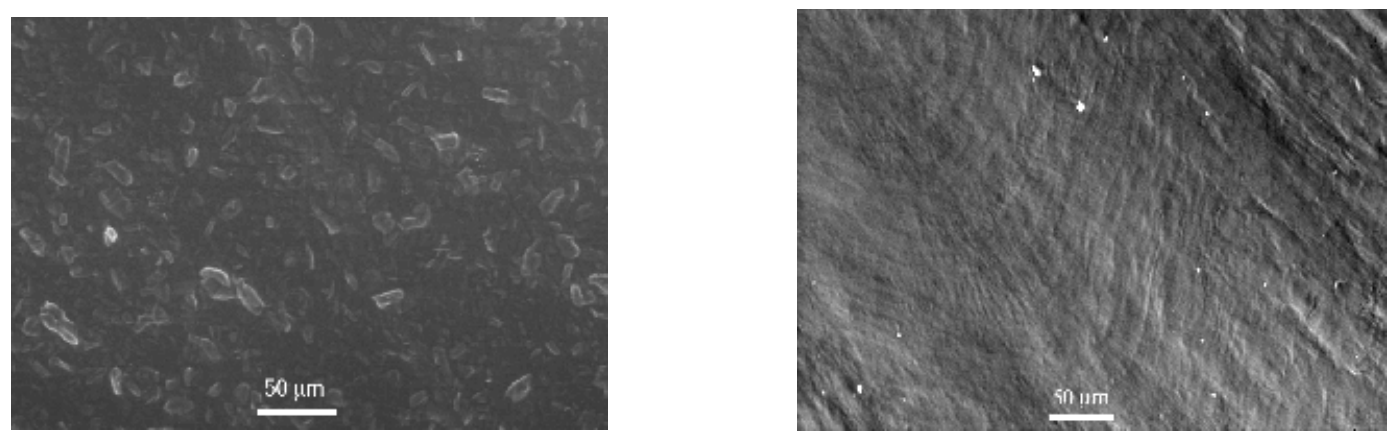

FIG. 3. ESEM micrographs of liquid crystalline phase texture a) minutes and b) hours after sample preparation (Beam energy was $10 \mathrm{keV}$, chamber pressure 4.1 Torr and sample temperature 275 $\mathrm{K}$ ). 